PROFESIONALES Y HERRAMIENTAS PARA EL DESARROLLO LOCAL Y SUS SINERGIAS TERRITORIALES. EVALUACIÓN Y PROPUESTAS DE FUTURO IX Coloquio Nacional de Desarrollo Local del GTDL-AGE 

ANTONIO MARTÍNEZ PUCHE, XAVIER AMAT MONTESINOS, ISABEL SANCHO CARBONELL y DANIEL SANCHIZ CASTAÑO (EDS.)

\section{PROFESIONALES Y HERRAMIENTAS PARA EL DESARROLLO LOCAL Y SUS SINERGIAS TERRITORIALES. EVALUACIÓN Y PROPUESTAS DE FUTURO}

IX Coloquio Nacional de Desarrollo Local del GTDL-AGE

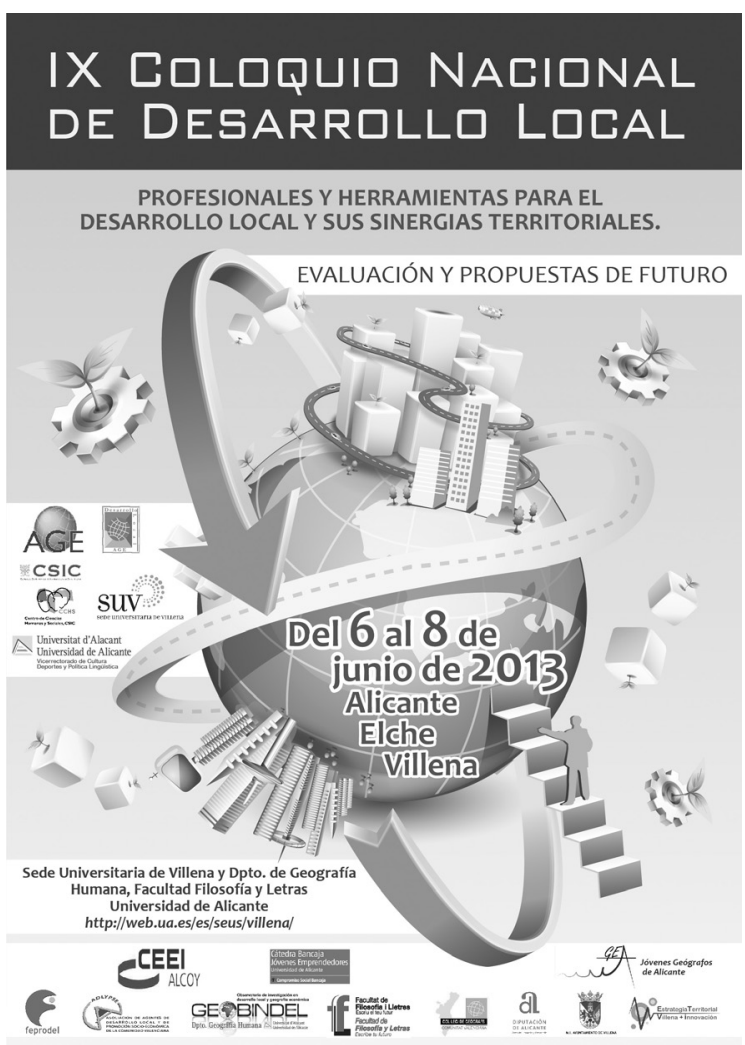


Este libro ha sido examinado y valorado por evaluadores ajenos a la Universidad de Alicante, con el fin de garantizar la calidad científica del mismo.

Publicacions de la Universitat d'Alacant

03690 Sant Vicent del Raspeig

Publicaciones@ua.es

http://publicaciones.ua.es

Telèfon: 965903480

(C) Antonio Martínez Puche, Xavier Amat Montesinos,

Isabel Sancho Carbonell y Daniel Sanchiz Castaño (eds.), 2016

(C) d'aquesta edició: Universitat d'Alacant

ISBN: 978-84-16724-00-0

Dipòsit legal: A 92-2016

Disseny de coberta: candela ink

Composició: Página Maestra (Miguel Ángel Sánchez Hernández)

Impressió i enquadernació: Guada Impresores

\section{unte \\ Unión de Editoriales
Universitarias Españolas \\ WWW.une.es
WWA}

Esta editorial es miembro de la UNE, cosa que garantiza la difusión y comercialización nacional y internacional de sus publicaciones.

Reservados todos los derechos. Cualquier forma de reproducción, distribución, comunicación pública o transformación de esta obra sólo puede ser realizada con la autorización de sus titulares, salvo excepción prevista por la ley. Diríjase a CEDRO (Centro Español de Derechos Repográficos, www.cedro.org) si necesita fotocopias o escanear algún fragmento de esta obra. 


\title{
LA RENTABILIDAD SOCIAL DE LAS EMPRESAS EN EL NUEVO MARCO SOCIOECONÓMICO. ESTUDIO DEL CASO DEL "CELLER LA MUNTANYA"
}

\author{
Rafael Montaner Brotons \\ Alumno del máster oficial Desarrollo Local e Innovación Territorial, UA \\ rafamontaner@yahoo.es
}

RESUMEN

El estudio en cuestión, está orientado a definir el concepto de Rentabilidad Social Empresarial (RSE), y cómo ésta afecta a un entorno rural concreto; en este caso, cómo se desarrollan estos conceptos con un efecto demostrativo, con empresas que aplican modelos socioeconómicos alternativos.

Para llegar a este punto, repasamos el cambio de tendencia del modelo empresarial, hacia una práctica socio-económica, basada en la proximidad, en el valor del entorno, en la producción local, en los parámetros de calidad ambiental, la formación y el fomento del empleo de calidad.

Existe una casuística en el entorno empresarial rural, que se convierte en objeto de estudio, puesto que estos conceptos no se han puesto en valor. Por lo tanto, tenemos la posibilidad de establecer o recuperar indicadores, para medir y cuantificar el concepto de Rentabilidad Social, y de esta manera ponderar de manera objetiva el beneficio socio-económico de estas empresas.

Además de un compromiso voluntario para y ante la sociedad, la RSE hay que entenderla como la implantación por la organización de la necesaria equidad en sus relaciones con los distintos grupos de interés (stakeholders) que confluyen en la Empresa: propietarios, empleados, clientes, proveedores, medios de comunicación, opinión pública, competidores, y la sociedad en su conjunto.

El efecto demostrativo de modelo aplicado utilizado, es la empresa "El Celler la Muntanya", la cual está trabajando en intentar definir estos conceptos, para así, conjuntamente con la administración, conseguir establecer una serie de incentivos (fiscales, económicos, etc.). Esta empresa fomenta la aplicación de la Economía del Bien Común, además de basar su sistema de producción de vinos, en pequeños minifundios vinícolas, que contribuyen a la conservación 
y preservación del entorno y el paisaje, asimismo realizando una importante labor social. Éste ámbito social de la empresa será de gran importancia, por lo que, para el avance de la investigación, parte fundamental es el trabajo de campo, para así conocer de primera mano la realidad de los cerca de 30 pequeños agricultores, que se benefician de este modelo de producción. De esta manera podremos plasmar, aunque de manera cualitativa, las impresiones, percepciones e inquietudes, de todos aquellos actores que se ven implicados de manera directa en la aplicación de modelos de RSE.

Por lo tanto, La RSE tiene su base en la existencia de principios y valores en alza, acentuados, por la situación socioeconómica global. Éstos son los que dan lugar a una actuación socialmente responsable, que se convierte en una necesidad para las empresas, la cual debe contemplar los intereses lícitos de todos los que se relacionan con la empresa, y supone además una respuesta a las nuevas demandas sociales que no estén recogidas en las leyes.

El objetivo de este trabajo de investigación, es el de poner en valor la necesidad de establecer una serie de indicadores para la medición de la Rentabilidad Social, además de intentar definir ésta estructura funcional, y, de esta manera convertirla en un modelo aplicable a otras empresas.

Seguramente el conjunto social acepte que el capitalismo sea la forma dominante de actividad económica; pero, a cambio, ha de exigir una profundización en el concepto de la "responsabilidad corporativa" incorporando los aspectos medioambientales y sociales.

Tenemos que aplicar un nuevo modelo de desarrollo local que se sustente en la cooperación entre los distintos agentes públicos y privados; con el objeto de potenciar, un "know how" basado en la Responsabilidad/Rentabilidad Social Empresarial.

Palabras clave: Rentabilidad Social Empresarial, Celler la Muntanya, $L a$ Economía del Bien Común, "QUOMMON TERRA".

\section{SOCIAL PROFITABILITY OF ENTERPRISES IN THE NEW SOCIOECONOMIC FRAMEWORK CASE STUDY OF "CELLER THE MUNTANYA"}

\section{AbSTRACT}

The study in question is aimed at defining the concept of Corporate Social Performance (CSR), and how it affects a particular rural environment; in this case, how these concepts with a demonstration that effects companies that apply alternative socio-economic models.

To reach this point, we reviewed the turnaround of the business model towards a socio-economic practice, based on proximity, on the value of the environment, local production, environmental quality parameters, training and promoting quality employment. 
There is a casuistry in the rural business environment that becomes an object of study, since these concepts have not been put at value. Therefore, we are able to set or retrieve indicators to measure and quantify the concept of social profitability, and thus objectively weigh the socio-economic benefit of these companies. In addition to a voluntary commitment to and for society, CSR must be understood as the implementation by the organization of the need for fairness in its relations with the different interest groups (stakeholders) that shaped the company: owners, employees, customers, suppliers, media, public, competitors, and society as a whole. The demonstration effect of the applied model, is the company "El Celler la Muntanya", which is working on trying to define these concepts, so that, together with management, obtain a set of incentives (fiscal, economic, etc.). This company promotes the application of the Economy of the Common Goods, in addition to its system of winemaking, small wineholdings, which contribute to the conservation and preservation of the environment and landscape, as well as performing an important social work. This social enterprise level will be important, therefore, for the advancement of research, a critical part is the field work in order to see firsthand the reality of about 30 small farmers who benefit from this production model. In this way we can capture, even qualitatively, impressions, perceptions and concerns of all those factors that are directly involved in the application of the CSR model.

Therefore, CSR is based on the existence of principles and values on the rise, accentuated by the global economic situation. These are leading to a socially responsible behavior, it becomes a necessity for businesses, which must consider the legitimate interests of all those connected with the company, and also represents a response to new social demands that are not collected by law.

The objective of this research is to value the need to establish a set of indicators to measure social returns, besides trying to define this functional structure, and thus make it a model applicable to other companies.

Surely the social group accept that capitalism is the dominant form of economic activity; but, in return, must demand a deepening of the concept of "corporate responsibility" incorporating the environmental and social aspects.

We need to apply a new local development model that relies on the cooperation between different public and private agents; in order to promote a "know-how" based on Responsibility / Corporate Social.

Key words: Corporate Social Performance, Celler la Muntanya, The Economy of the Common Good, "QUOMMON TERRA". 


\section{INTRODUCCIÓN}

La finalidad de este trabajo, está orientada a definir el concepto de Rentabilidad Social Empresarial (RSE), y de cómo ésta afecta a un entorno rural concreto. En este caso, estudiaremos cómo se desarrollan estos conceptos con un efecto demostrativo, con empresas que aplican modelos socioeconómicos alternativos.

Para llegar a este punto, debemos entender el cambio de tendencia del modelo empresarial hacia una práctica socio-económica, basada en la proximidad, en el valor del entorno, en la producción local, en los parámetros de calidad ambiental, la formación y el fomento del empleo de calidad.

En su libro "La Economía del Bien Común", (Felber, 2012 p. 51), se pregunta: ¿Por qué seguimos confundiendo el "éxito" de una empresa con su beneficio financiero? Ya que el beneficio de una empresa sólo ofrece información de cómo se sirve a sí misma, pero no de cómo sirve a la sociedad o al entorno en el que opera.

Así pues existe una casuística en el entorno empresarial, que se convierte en objeto de estudio, puesto que estos conceptos no se han puesto en valor. Por lo tanto, la pretensión, es la de establecer o recuperar indicadores, para medir y cuantificar el concepto de Rentabilidad Social, y de esta manera ponderar de manera objetiva el beneficio socio-económico de estas empresas.

\section{LA RENTABILIDAD SOCIAL EMPRESARIAL COMO HERRAMIENTA}

Además de un compromiso voluntario para y ante la sociedad, la RSE hay que entenderla como la implantación por la organización de la necesaria equidad en sus relaciones con los distintos grupos de interés (stakeholders) que confluyen en la Empresa: propietarios, empleados, clientes, proveedores, medios de comunicación, opinión pública, competidores, y la sociedad en su conjunto (García, 2009).

Otro aspecto que no podremos obviar, será cómo estas buenas prácticas, afectan y se ven afectadas por el entorno macro y micro de esta empresa; por lo que la RSE, deberá ir asociada a una hoja de ruta, unas directrices y unos indicadores, que nos ayuden a definir a una empresa como socialmente responsable, para que, este saber hacer, revierta en el empresario de manera tangible, por lo que posiblemente, tengamos que conjugar la rentabilidad social, con la rentabilidad empresarial o económica, huyendo de las acepciones peyorativas del término; ya que la competencia/competitividad ha permitido optimizar recursos de la economía; ha contribuido al crecimiento, y por tanto ha mejorado nuestros estándares de vida (Crespo, 2012 p. 128). De esta manera, lo más sensato sería evitar ahondar en conceptos macroeconómicos para aportar soluciones a nuestro entorno empresarial, económico y comercial; y hacerlo desde la práctica del desarrollo local conjugado con la puesta en valor por parte del sector privado, de políticas que fomentan la Rentabilidad Social Empresarial (RSE). 
La Responsabilidad Social Corporativa, presenta por tanto gran relevancia como factor clave de la estrategia empresarial de muchas grandes empresas a la hora de alcanzar el objetivo de toda actividad económica, el cual no debemos obviar, es la maximización del beneficio. Así pues, para la mayoría de las empresas, realizar una gestión óptima de la Responsabilidad Social Corporativa supone una ventaja competitiva muy importante y un factor estratégico de éxito empresarial.

Cabe destacar, que no se cuestiona la necesidad de generar valor añadido de las empresas, de proporcionar bienes y servicios al mercado, de tener clientes y de maximizar beneficios; pero sí se debe poner de manifiesto que éstos conceptos quedan obsoletos a la hora de generar valor añadido y ventaja competitiva, por lo que resultan hoy totalmente limitados.

Seguramente el conjunto social acepte que el capitalismo sea la forma dominante de actividad económica; pero, a cambio, ha de exigir una profundización en el concepto de la "responsabilidad corporativa" incorporando los aspectos medioambientales y sociales (Gallizo, 2006). Además, la acumulación de valores materiales es el fin del capitalismo y pronto sobrepasa lo que sería un efecto secundario razonable, satisfacer las necesidades básicas, doblegando otros valores tales como la calidad de las relaciones y el cuidado del medio ambiente, tiempo de bienestar, creatividad, autonomía (Felber, 2012 p. 44).

\subsection{Responsabilidad Social Corporativa VS. Rentabilidad Social Empresarial}

De esta manera, la RSC como concepto clásico, ha permanecido integrada en la cadena de valor de las actividades económicas, como factor estratégico, y herramienta de gestión y control, convertida en un elemento de marketing para la mejora de la imagen corporativa, de manera que más allá de la actuación de RSC, no existía o existe una cultura o unos valores de sensibilización implantados en la empresa; lo cual nos lleva a cuestionarnos si las empresas que aplican políticas de RSC, son realmente socialmente responsables, ya que la utilización de estos métodos ha permitido dotar de rentabilidad económica a la responsabilidad social, sin prejuicio de la rentabilidad social de las actividades económicas llevadas a cabo en un entorno determinado.

Llegados a este punto, y como más adelante desarrollaremos, debemos señalar, que la intención de este estudio, es la de intentar formular las directrices de futuras líneas de investigación, intentando huir de la descripción de la metodología de la RC, ahondando en debates terminológicos; por lo que de ahora en adelante, intentaremos utilizar el concepto de Rentabilidad Social Empresarial (RSE), y buscaremos la utilidad de la misma, para que las empresas consideradas socialmente responsables, puedan ver recompensado su know-how y la sensibilización con el entorno y el medioambiente, a través de incentivos fiscales, programas formativos, promoción, reducción de costes, etc. 
Tal como apunta Felber (FELBER, C., 2012; 52), muchas empresas, en especial las empresas globales y las firmas de marca que protegen su reputación, en los últimos años han tomado en cuenta las críticas a su unilateral y desmedido afán por el beneficio y han reaccionado. Mediante etiquetas en sus productos (agricultura ecológica, comercio justo), sistemas de gestión ambiental (EMAS, ISO), sistemas de gestión de calidad (EFQM, el Cuadro de Mando Integral), códigos de conducta e informes de sostenibilidad (GRI) quieren demostrar que también se preocupan por el bien común y actúan de forma "socialmente responsable". El problema, es que estos instrumentos de Responsabilidad Social Corporativa no son obligatorios ni se controlan desde ninguna autoridad legal.

\subsection{Alcance de la RSE}

Existe un debate importante a nivel económico, social y político, sobre el alcance de la RSE en la empresa, y por lo tanto, surge la duda de si se trata de una herramienta de gestión empresarial para la mejora de la imagen corporativa y la maximización de los beneficios, o si por otra parte, esta se debe implementar en la empresa cómo un modelo de gestión de los procesos que en ella se llevan a cabo, de manera que la actividad económica, social y responsable tenga su reflejo en el territorio.

Según (Muñoz, 2011), la RSC debe integrar, además del cumplimiento estricto de las obligaciones legales vigentes, la integración voluntaria en el gobierno, estrategia y gestión de las empresas en las políticas y procedimientos sociales, laborales, económicos, medioambientales, de respeto a los derechos humanos, etc. que surgen de la relación y el diálogo transparente con sus grupos de interés, por lo que consideraremos más adecuado hablar de Rentabilidad Social Empresarial (RSE).

Así pues, podemos entender que el objetivo futuro, ha de ser el de instaurar una serie de indicadores para la medición de la Rentabilidad Social, además de intentar definir ésta estructura funcional, y, de esta manera convertirla en un modelo aplicable/adaptable a otras empresas.

\subsection{La RSE como herramienta para generar Valor Añadido}

Por lo tanto, se impone como necesario para el desarrollo de las medianas y pequeñas empresas, el aplicar metodologías que conduzcan a la obtención de un valor añadido o elemento diferenciador, que les ayude a destacar a través de una ventaja competitiva. Pero, nos debemos preguntar si en este caso basta con la utilización de la RSE cómo herramienta estratégica de marketing, o si las pymes deben trabajar en desarrollar una actividad socialmente responsable con el entorno en el que operan.

Se trata, en definitiva, de conjugar los objetivos económicos, sociales y medioambientales y conciliar, evidentemente, los fines que persigue la empre- 
sa con los de la sociedad. Según (Felber, 2012), El marco de incentivos para los individuos activos en la economía tiene que cambiar radicalmente de la búsqueda de beneficios y la competencia a la búsqueda del bien común y la cooperación.

Por lo tanto, una empresa se considerará económica, medioambiental y socialmente responsable cuando responda satisfactoriamente a las expectativas que sobre su funcionamiento tienen los diversos stakeholders y se responsabilice de las consecuencias y los impactos que se deriven de sus acciones, y por tanto, sus actividades proporcionen una Rentabilidad Social al entorno en el que opera, a nivel territorial, social, ambiental, económico y político.

El principal inconveniente, de acuñar esta nomenclatura, estará en poder definir y acotar los conceptos citados anteriormente, y en qué grado son tratados por la empresa socialmente responsable; por lo que como señalábamos, surgirá la necesidad de establecer indicadores e incentivos que nos ayuden a cuantificar conceptos aparentemente subjetivos, como la conservación del territorio, la concienciación medioambiental, la formación, el fomento del empleo, los parámetros de calidad ambiental, el modelo económico aplicado, etc.

Por lo tanto, a la hora de definir un proceso de gestión adecuado de la Rentabilidad Social Empresarial, debemos entender este concepto en sus diversas acepciones, y situar a la empresa como una entidad responsable con el entorno, con los stakeholders, y con las instituciones. (Olcese, et al., 2008 p. 188).

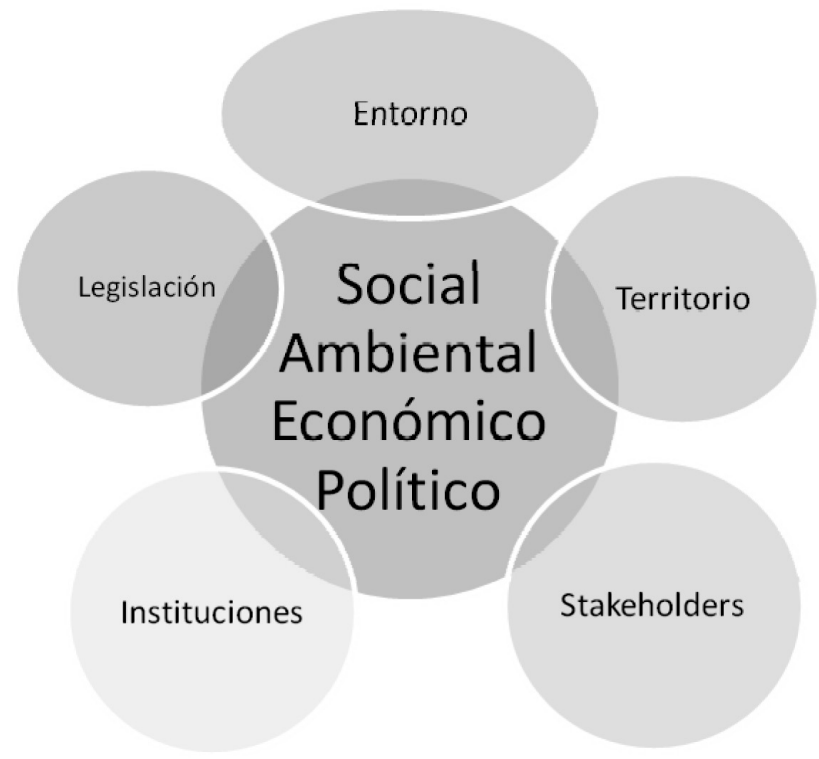

Figura 1. Esquema Responsabilidad Social Corporativa

Fuente: Elaboración Propia 
Llegados a este punto; deberemos trabajar en definir el Índice de Responsabilidad Social (IRS), para poder identificar aquellas actividades que definen una empresa como socialmente responsable.

Es decir, surge la necesidad de establecer indicadores para medir la RSE, y de esta forma poder determinar en qué grado las empresas son socialmente responsables, y de qué manera estas actuaciones se ven favorecidas mediante políticas compensatorias, huyendo de las actuales medidas coercitivas que gravan las actuaciones lesivas.

La aplicación de la RSE tiene su base en la existencia de principios y valores, implantados en todos los niveles de la empresa, y asumidos como parte del proceso productivo, ya que afectan directamente a la imagen corporativa. Éstos son los que dan lugar a una actuación socialmente responsable, que debe contemplar los intereses lícitos de todos los que se relacionan con la empresa, y supone además una respuesta a las nuevas demandas sociales que no estén recogidas en las leyes (García, 2009: 156).

Por su parte, el artículo 39 de la Ley 2/2011 de 4 de marzo, de Economía Sostenible, en su párrafo primero, señala que, con el objetivo de incentivar a las empresas, organizaciones e instituciones públicas o privadas, especialmente a las pequeñas y medianas empresas individuales, a incorporar o desarrollar políticas de responsabilidad social, difundiendo su conocimiento y las mejores prácticas existentes y estimulando el estudio y análisis sobre los efectos en materia de competitividad empresarial de las políticas de responsabilidad social.

En los ámbitos señalados, el Real Decreto 343/2012, de 10 de febrero, establece que la Dirección General del Trabajo Autónomo, de la Economía Social y de la Responsabilidad Social de las Empresas tiene entre otras funciones, la promoción de la Responsabilidad Social de las Empresas, en particular de las pequeñas y medianas, así como la realización de cualquier otra actividad que se le encomiende en dicho ámbito de actuación; y el diseño, gestión y seguimiento de programas y ayudas para la difusión y fomento de la Responsabilidad Social de las Empresas.

En este sentido; tal como indica Felber, existen ya "incentivos" apropiados, tan solo se tienen que utilizar consecuentemente para favorecer la responsabilidad de las empresas, por ejemplo:

- Disminución de Impuestos.

- Aranceles más bajos.

- Facilidad de acceso a créditos bancarios mediante avales.

- Prioridad en la compra pública y en la adjudicación de contratos.

- Cooperación con universidades públicas en investigación.

- Ayudas directas. 


\section{Efecto demostrativo: "El Celler la Muntanya"}

El ejemplo demostrativo es el Celler la Muntanya ${ }^{1}$; una empresa nacida en 2004 y dedicada a la producción y venta de vinos y aceites de elevada calidad, ubicada en Muro de Alcoy, en la comarca del Comtat (Alicante), la cual está trabajando en intentar definir conceptos anteriormente citados, para así, conjuntamente con la administración, conseguir establecer una serie de incentivos (fiscales, económicos, etc.).

Esta empresa fomenta la aplicación de la Economía del Bien Común (Felber, 2012), además de basar su sistema de producción de vinos, en pequeños minifundios vinícolas, característicos de la estructura física de las zonas montañosas del Comtat, que contribuyen a la conservación y preservación del entorno y el paisaje, asimismo realizando una importante labor social.

La empresa se abastece de uva mediante la iniciativa Microviña ${ }^{\circledR}$. Se trata de un proyecto que permite la participación de terceros mediante la conducción de pequeños viñedos, nuevos y recuperados, consiguiendo así una múltiple rentabilidad económica, social, cultural, y ambiental de las explotaciones minifundistas. La implementación del proyecto Microviña ${ }^{\circledR}$ se está realizando actualmente en municipios como Agres, Alcocer, Alcoi, Beniarrés, Gaianes, Gorga, Benifallim, Banyeres, Biar, Cocentaina, Muro y Tibi, entre otros, y ha permitido la recuperación de las superficies dedicadas al cultivo de la vid a través de más de 30 microviñas (Tormo, 2011: 83).

Este proyecto proporciona a los propietarios de pequeños minifundios, la posibilidad de implantar el cultivo de la vid en sus tierras, obteniendo a cambio, asesoramiento, conocimientos, y una contraprestación por el producto final, fomentando así la actividad agraria en la zona; contribuyendo a la dinamización económica y sobre todo, a la conservación del entorno y el paisaje mediante la recuperación de superficies aptas para el cultivo vinícola.

Este ámbito social de la empresa será de gran importancia, por lo que, para el avance de futuras investigaciones, será fundamental el trabajo de campo, para así conocer de primera mano la realidad de los cerca de 30 pequeños agricultores, que se benefician de este modelo de producción, y de esta manera plasmar, aunque de manera cualitativa, las impresiones, percepciones e inquietudes, de todos aquellos actores que se ven implicados de manera directa en la aplicación de modelos de RSE.

\subsection{Proyecto "QUOMMON"}

Con todo esto; una de las principales iniciativas nacidas desde el "Celler la Muntanya", és el proyecto "QUOMMON TERRA", insertado dentro del programa PROGRESS de la Unión Europea. Esta iniciativa responde a la nece-

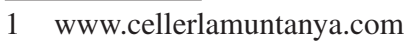


sidad de crear un programa que acoja y reúna el "Know how" del sistema empresarial rural; y con la finalidad de aplicar iniciativas del Bien común en el sistema económico del entorno en el que opera.

\subsubsection{Objetivos Generales del Programa Progress ${ }^{2}$}

PROGRESS es el programa de la UE para el empleo y la solidaridad social, creado para dar apoyo financiero para la consecución de los objetivos de la UE en empleo, asuntos sociales e igualdad de oportunidades.

Sus líneas de trabajo principales son:

- La aplicación de la Estrategia Europea de Empleo (artículo 1)

- La aplicación del método de coordinación abierta en materia de protección social e inclusión (sección 2)

- La mejora del entorno y las condiciones para la salud y seguridad en el Trabajo y la conciliación de la vida laboral y familiar (artículo 3)

- La aplicación efectiva del principio de no discriminación y la promoción de su integración en todas las políticas de la UE (artículo 4)

- La aplicación efectiva del principio de la igualdad de género y la promoción de su integración en todas las políticas de la UE (artículo 5).

\subsubsection{Intervención en el Ecosistema Empresarial de l'Alcoià y el Comtat}

Dada la existencia de esta herramienta (dotación económica para llevar a cabo experimentos sociales), así como las sinergias que en las comarcas de l'Alcoià y el Comtat se han generado alrededor del Proyecto Microviña, tanto a nivel de reflexiones como de actividades concretas, y dada la repercusión en positivo que han tenido en diferentes medios de comunicación locales, comunitarios y nacionales, un equipo de personas impulsoras, plantean a la recién creada "Mancomunidad de l'Alcoià i el Comtat" la posibilidad de poner en marcha un experimento social que pueda evaluar y substanciar la idea de que es posible configurar un modelo económico fundamentado en la tierra donde operan nuestras empresas, en donde se valoren otras rentabilidades más allá de la económica, sin renunciar a ella. Lo que actualmente se denomina Economía de abajo a arriba "bottom up" para el Bien Común. La cuestión que se plantea es: El aumento de la rentabilidad social y su medición, ¿Puede fomentar el empleo entre nuestros jóvenes?

2 Información disponible en el documento "Programa de Aplicación del Bien Común en el Ecosistema Empresarial Rural", desarrollado por el equipo impulsor del proyecto QUOMMON 22/01/2013 www.quommon.org 


\subsubsection{Idea de Proyecto}

Se pretende proporcionar una formulación específica y una fuerte evidencia experimental basada en un modelo de desarrollo económico territorial, con la cual se busca una acción de cambio en las políticas Europeas en relación con la tributación y beneficios fiscales de los impuestos, así como establecer bonificaciones para las empresas que emplean a jóvenes desempleados, entre otras.

Así mismo, el proyecto establece cuatro objetivos específicos:

1. Establecer y calcular, a partir de los resultados obtenidos por dicho mecanismo, un índice de bienestar social.

2. Diseñar un mecanismo basado en un conjunto de métricas que definan con precisión el bienestar social generado por las empresas, teniendo en cuenta que éstas son un conjunto de actores (stakeholders): propietarios, asalariados, financiadores, proveedores, clientes, entorno social, y medioambiente.

3. Demostrar que dicho índice es representativo de la rentabilidad social sin menoscabar la rentabilidad financiera.

4. Desarrollar un experimento social acotado a un entorno de ámbito comarcal rural-periférico, el cual confirme que las empresas que presentan mayores valores en dicho índice, impactan de manera más positiva en la generación de empleo, desarrollo y dinamización económica.

Para alcanzar estos objetivos el equipo impulsor considera que el entorno rural, entorno de cercanía, es el escenario adecuado para medir la eficacia del proyecto. Empresas de talla humana, que hoy en día son las que más sufren la crisis y son las que fomentan la actividad en su entorno próximo. Parte importante será también la involucración directa de Ayuntamientos, organismos educativos y ciudadanos que buscan qué hacer para que los pueblos recuperen el atractivo de una vida normal, en la que se cuente con posibilidades de crecimiento económico. Así pues, en colaboración con Ayuntamientos y organismos públicos que acepten el proyecto, se seleccionará un conjunto de empresas que se presten al ejercicio de aplicar el mecanismo que permita crear el índice del bienestar, y así comparar la evolución que dichas empresas tendrán con otro conjunto de empresas que no apliquen el índice del bienestar y todo lo que dicho índice comporta.

Siendo de mayor utilidad otro tipo de indicadores, en detrimento de los anteriormente citados, apropiados por las grandes empresas para mejorar su imagen, para empresas como el Celler la Muntanya que operan en un entorno rural y apuestan por el consumo de proximidad, tales como:

- La utilidad de los productos/servicios

- Las condiciones laborales

- Si la empresa produce de manera ecológica

- La relación con el grupo de stakeholders 
- El modelo socioeconómico aplicado

- La distribución del beneficio

- La solidaridad de la empresa

- La equidad en las condiciones laborales

- La apuesta por la conservación del entorno

- El fomento de la cultura del territorio

- La gestión en la toma de decisiones

\section{Conclusiones}

Según las premisas anteriormente expuestas, queda reflejado, el carácter utilitarista de la Responsabilidad Social en el mundo económico-empresarial; por lo que habrá que trabajar en definir estos conceptos, y el uso que hacen de ellos las empresas. Para ello, habrá que empezar a trabajar desde lo local, desde las experiencias cercanas, que operan en un territorio determinado que forma parte de su "Know-how", en dónde los recursos endógenos se convierten en un valor añadido y las relaciones interprofesionales forman parte de la cadena de valor; constituyendo así, un sistema socialmente responsable, capaz de potenciar valores y buenas prácticas en toda la cadena del proceso productivo, desde el proveedor inicial hasta el cliente final. Quedando latente la necesidad de potenciar o premiar estas políticas de Desarrollo Local llevadas a cabo desde la perspectiva empresarial rural, capaz de fomentar la promoción económica, el empleo, la igualdad de géneros, la conservación del medioambiente, la custodia y puesta en valor del territorio, etc.

Tenemos que trabajar pues, en aplicar un nuevo modelo de desarrollo local que se sustente en la cooperación entre los distintos agentes públicos y privados, entendida esta, como una colaboración que permita alcanzar un fin común, (Tormo, 2012 p. 80). Finalmente, será fundamental el contacto con la Administración y su adaptación a la jurisdicción, para intentar definir beneficios fiscales o económicos para incentivar el desarrollo de éstas buenas prácticas.

BIBLIOGRAFÍA

CRESPO, J. (2012): Las dos próximas recesiones, Barcelona : Grupo Planeta, $128 \mathrm{p}$.

FELBER, C. (2012): La Economía del Bien Común, Viena : DEUSTO, pp. 51-52.

GALLIZO, J. (2006): Responsabilidad social e información medioambiental en la empresa", Madrid : Aeca monografías.

GARCÍA, J. (2009): "Aproximación a la Responsabilidad Social de la Empresa: reflexiones y propuestas". - Madrid : Fundación Mapfre. - pp. 156-157.

MUÑOZ, L. (2011): "Gestión de la responsabilidad social corporativa en la PYME". 
OLCESE, A. y RODRÍGUEZ, M. (2008): "Manual de la Empresa Responsable y Sostenible. Conceptos y herramientas de la Responsabilidad Social Corporativa o de la Empresa". - Madrid : MCGRAW-HILL / INTERAMERICANA DE ESPAÑA, S.A. - p. 188.

TORMO, J. (2011): "Mariola: sistema productivo y estrategia territorial. - San Vicente del Raspeig : Publicaciones de la Universidad de Alicante". - p. 83. TORMO, J. (2012): "Sin cooperación no habrá desarrollo local” en Martínez Puche, A. y Calvo Palomares, R. (coord.), Valoraciones técnicas y repercusiones territoriales sobre el ejercicio profesional del desarrollo local, Editorial Germanía, Alzira (Valencia), pp. 79-86. 